\title{
Antireflection Coating at Metamaterial Waveguide Structure by Using Superlattices (LANS)
}

\author{
H. M. Mousa \\ Physics Department, Al Azhar University, Gaza, Palestinian \\ Email: h.mousa@alazhar-gaza.edu.ps
}

Received 19 March 2014; revised 18 April 2014; accepted 15 May 2014

Copyright (C) 2014 by author and Scientific Research Publishing Inc.

This work is licensed under the Creative Commons Attribution International License (CC BY).

http://creativecommons.org/licenses/by/4.0/

(c) (i) Open Access

\section{Abstract}

The characteristics of electromagnetic wave reflection and transmission by multilayered structures consisting of a pair of left-handed material (LHM) and superlattices (LANS) slabs inserted between two semi-infinite dielectric media are investigated for photovoltaic and solar energy applications. Maxwell's equations are used to determine the electric and magnetic fields of the perpendicular polarized wave incident at each layer. Snell's law is applied and the boundary conditions are imposed at each layer interface to calculate the reflected and transmitted coefficients of the structure. The reflected, transmitted powers are determined using these coefficients by a recursive method. The reflected and transmitted powers are computed in both visible and microwave spectral band with the appropriate LHM for each band and appropriate location of LANS in the structure. They are illustrated as a function of the incident wavelength, angle of incidence, magnetic fraction of LANS and thickness of the slabs with the emphasis on the appropriate refractive indices. I found that, zero reflectance and maximum transmittance of the incident powers are achieved for visible spectral band at a single frequency if LHM and LANS have the same refractive index of opposite signs with the same width and more magnetic material of LANS while the reflected power is zero for less magnetic material of LANS in the microwave spectral band which realizes antireflection coating in this structure.

\section{Keywords}

Angle of Incidence, Magnetic Fraction, Metamaterial, Microwave Band, Reflection, Transmission, Superlattices, Visible Band

\section{Introduction}

In many applications, reflection is undesirable and causes insertion losses, for example. It is well known that the 
application of one or more antireflection coating (ARC) layer on the front surface of the photovoltaic cells and optoelectronic devices (Lasers, IR diodes, etc.) reduces the amounted reflection of the incident light, which improves the device performance [1]-[3]. Antireflection coatings require a particular refractive index and quarter wavelength thickness where it operates by overcoming the mis-match between intrinsic impedances of two media. This approach is scalable over a wide spectral range from microwave to far infrared [4]. There have been a few efforts to develop antireflection coatings at $\mathrm{THz}$ frequencies by using dielectric meta-materials or lefthanded materials (LHM). LHM have both negative permittivity and permeability and consequently have negative index of refraction [5] [6] and are able to match media impedances. The theory of antireflection coating is examined by many authors [7] [8]. The matrix method [9] [10] is usually employed for calculation of reflection coefficient. Chen et al. [4] have presented approach of metamaterial antireflection.

It reduces the reflection and enhances transmission near a specifically frequency over a wide range of incidence angles for both TE and TM polarizations, Bouhafs et al. [3] have made a theoretical study of the antireflection coatings on silicon solar cells. Cory et al. [11] have analyzed the reflection and transmission characteristics of a multilayered structure consisting of metamaterials and dielectric slabs. In this paper, I investigate the reflection and transmission properties of a superlattices (LANS)-metamaterial (LHM)-dielectric multilayered structure. Since antireflection coating is formed by two slabs of the same thickness and of opposite refractive indices, a pair of LHM and LANS materials is situated between two semi-infinite dielectric media which are considered right handed materials (RHM) of positive refractive index. The superlattice is a lateral anti-ferromagnetic/nonmagnetic (LANS) such as $f_{e} f_{2} / z_{n} f_{2}$. LANS are described with an effective medium theory. Such description is valid when the wave lengths of the excitations are much longer than the superlattice period where $k L \ll 1$, where $k$ is the magnitude of the wave vector and $L=L_{1}+L_{2}$, is the period of the superlattice, $L_{1}$ and $L_{2}$ are the thickness of the anti-ferromagnetic layers and non-magnetic layers ,respectively [5] [12]. $f_{1}=L_{1} / L$ and $f_{2}=L_{2} / L$. In the theory the electric and magnetic fields of the incident waves are determined in each region by Maxwell's equations. Then Snell's law is applied and the boundary conditions are imposed at each interface to obtain the reflection and transmission coefficients. The reflected and transmitted powers of the structure are presented in terms of these coefficients. In the numerical analysis, a recursive method [13] is used to calculate the reflected, transmitted powers as a function of incident wavelength, angle of incidence, layer thickness and magnetic fraction of (LANS). The calculations are performed for electromagnetic radiations in both the visible and microwave bands for a single wavelength by selecting the optimum refractive indices of LHM and RHM in both bands. The suitable LHM in each band is selected. The conservation law of energy is checked and satisfied.

\section{Theory}

Consider LHM and LANS of electric permittivity and magnetic permeability $\left(\varepsilon_{h}, \mu_{h}\right)$ and $\left(\varepsilon_{\perp}, \mu^{e}\right)$ respectively embedded between two semi-infinite dielectric media of permeability and permittivity as $\left(\mu_{1}, \varepsilon_{1}\right)$ and $\left(\mu_{4}, \varepsilon_{4}\right)$. A perpendicular polarized wave is incident on the structure at $Y=0$ at angle $\theta$ relative to the normal to the boundary as depicted in Figure 1.

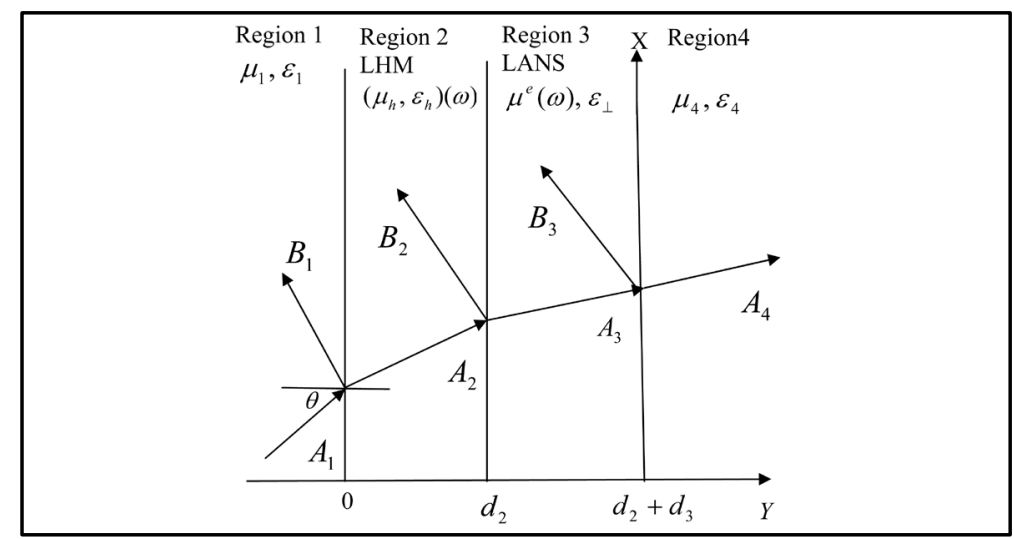

Figure 1. Wave propagation through a structure consisting of LHM and LANS materials inserted between two semi-infinite dielectric media. 
Introducing the effective medium theory, the magnetic permeability of the LANS [5] [12] which is described as a single effective medium, can be written as:

$$
\mu^{e}=\left(\begin{array}{ccc}
\mu_{x x} & i \mu_{x y} & 0 \\
-i \mu_{x y} & \mu_{y y} & 0 \\
0 & 0 & 1
\end{array}\right)
$$

with

$$
\begin{aligned}
& \mu_{x x}=\left[1 /\left(f_{1}+f_{2} \mu\right)\right]\left[\left(f_{1}^{2}+f_{2}^{2}\right) \mu+f_{1} f_{2}\left(1+\mu^{2}-\mu_{\perp}^{2}\right)\right] \\
& \mu_{y y}=\mu /\left(f_{1}+f_{2} \mu\right), \quad \mu_{x y}=f_{1} \mu_{\perp} /\left(f_{1}+f_{2} \mu\right)
\end{aligned}
$$

where the expressions of $\mu$ and $\mu_{\perp}$ are [5] [12]:

$$
\begin{gathered}
\mu=1+\omega_{a} \omega_{m}\left\{\left[\omega_{r}^{2}-\left(\omega_{0}-\omega\right)^{2}\right]^{-1}+\left[\omega_{r}^{2}-\left(\omega_{0}+\omega\right)^{2}\right]^{-1}\right\} \\
\mu_{\perp}=\omega_{a} \omega_{m}\left\{\left[\omega_{r}^{2}-\left(\omega_{0}-\omega\right)^{2}\right]^{-1}+\left[\omega_{r}^{2}-\left(\omega_{0}+\omega\right)^{2}\right]^{-1}\right\}
\end{gathered}
$$

with

$$
\begin{gathered}
\omega_{m}=4 \pi \gamma m_{0}, \quad \omega_{a}=\gamma H_{a}, \quad \omega_{0}=\gamma H_{0} \\
\omega_{r}=\gamma \sqrt{2 H_{a} H_{e}+H_{a}^{2}}
\end{gathered}
$$

$H_{a}$ represents an isotropy field, $H_{e}$ the exchange field, and $\gamma$ the gyromagnetic ratio. $m_{0}$ is the sublattice magnetization. The magnetic field of the superlattice is $H_{0}$. The effective dielectric function of (LANS) is expressed by [5] [13] as:

$$
\varepsilon_{\perp}=\varepsilon_{1} f_{1}+\varepsilon_{2} f_{2}
$$

The electric and magnetic field vectors for TE waves propagating along $\mathrm{x}$-axis with angular frequency $\omega$ are defined as:

$$
\begin{aligned}
& E=\left[0,0, E_{z}(\omega, y)\right] \exp i\left(k_{l x} x-\omega t\right) \\
& \vec{H}=\left[H_{x}(\omega, y), H_{y}(\omega, y), 0\right] \exp i\left(k_{l x} x-\omega t\right)
\end{aligned}
$$

The electric field in each region is [14], [15]:

$$
E_{l z}=\left(A_{l} \mathrm{e}^{i k_{l y} y}+B_{l} \mathrm{e}^{-i k_{l y} y}\right)
$$

where $A_{l}$ and $B_{l}$ are the amplitude of forward and backward travelling waves in the region of order $(l=1,2,3,4), \quad k_{l}=\frac{n_{l} \omega}{c}$ is the wave vector inside the material and $n_{l}$ is the refractive index of it.

\section{In Superlattice (LANS) Region}

The curl Maxwell's equations are [12]:

$$
\begin{aligned}
& \frac{\partial E_{3 z}}{\partial y}=-i \omega \mu_{o}\left(\mu_{x x} H_{3 x}-i \mu_{x y} H_{3 y}\right) \\
& k_{3 x} E_{3 z}=-\omega \mu_{o}\left(-i \mu_{x y} H_{3 x}+\mu_{y y} H_{3 y}\right) \\
& i k_{3 x} H_{3 y}-\frac{\partial H_{3 x}}{\partial y}=-i \omega \varepsilon_{o} \varepsilon_{\perp} E_{3 z}
\end{aligned}
$$

By these equations 


$$
\left.H_{3 x}=\frac{1}{i \omega \mu_{0} \mu_{v}}\left[\frac{\partial E_{3 z}}{\partial y}-\frac{k_{3 x}}{\mu_{y y}} \mu_{x y} E_{3 z}\right)\right]
$$

where $\mu_{v}=\left(\frac{\mu_{x x} \mu_{y y}-\mu_{x y}^{2}}{\mu_{y y}}\right)$ is the Voigt permeability.

By Maxwell's equation $\nabla x \boldsymbol{E}_{l}=i \omega \mu_{l} \boldsymbol{H}_{l}$, the magnetic field in the other regions of order $(l=1,2,4)$ is:

$$
\boldsymbol{H}_{l x}=\frac{1}{\mu_{l} \omega}\left(-A_{l} k_{l y} \mathrm{e}^{i k_{l y} y}+B_{l} k_{l y} \mathrm{e}^{-i k_{l y} y}\right)
$$

Matching the boundary conditions at each layer interface, where at $y=0, E_{1 z}=E_{2 z}$ and $H_{1 x}=H_{2 x}$ and so on yields six equations with six unknown parameters as:

$$
\begin{gathered}
A_{1}+B_{1}=A_{2}+B_{2} \\
\frac{k_{1 y}}{\mu_{1}}\left(A_{1}-B_{1}\right)=\frac{k_{2 y}}{\mu_{h}}\left(A_{2}-B_{2}\right) \\
A_{2} \mathrm{e}^{i k_{2 y} d_{2}}+B_{2} \mathrm{e}^{-i k_{2 y} d_{2}}=A_{3} \mathrm{e}^{i k_{3 y} d_{2}}+B_{3} \mathrm{e}^{-i k_{3 y} d_{2}} \\
\frac{k_{2 y}}{\mu_{h}}\left(A_{2} \mathrm{e}^{i k_{2 y} d_{2}}-B_{2} \mathrm{e}^{-i k_{2 y} d_{2}}\right)=\left(\frac{k_{3 y} \mu_{y y}+k_{3 x} \mu_{x y}}{\mu_{v} \mu_{y y}}\right)\left(A_{3} \mathrm{e}^{i k_{3 y} d_{2}}-B_{3} \mathrm{e}^{-i k_{3 y} d_{2}}\right) \\
A_{3} \mathrm{e}^{i k_{2 y}\left(d_{2}+d_{3}\right)}+B_{3} \mathrm{e}^{-i k_{3 y}\left(d_{2}+d_{3}\right)}=A_{4} \mathrm{e}^{i k_{4 y}\left(d_{2}+d_{3}\right)} \\
\left(\frac{k_{3 y} \mu_{y y}+k_{3 x} \mu_{x y}}{\mu_{v} \mu_{y y}}\right)\left(A_{3} \mathrm{e}^{i k_{3 y}\left(d_{2}+d_{3}\right)}-B_{3} \mathrm{e}^{-i k_{3 y}\left(d_{2}+d_{3}\right)}\right)=\frac{k_{4 y}}{\mu_{4}} A_{4} \mathrm{e}^{i k_{4 y}\left(d_{2}+d_{3}\right)}
\end{gathered}
$$

According to Snell's law $k_{1 x}=k_{2 x}=k_{3 x}=k_{4 x}, k_{l y}=\frac{\omega}{c} \sqrt{n_{l}^{2}-n_{1}^{2} \sin ^{2}(\theta)}$ with

$$
n_{3}=\sqrt{\frac{\mu_{y y}}{\mu_{x x}} \varepsilon_{\perp} \mu_{v}}, \quad n_{2}=\sqrt{\varepsilon_{h} \mu_{h}}
$$

For TE polarized light, at the first interface, the Fresnel coefficient (interface reflection and transmission $(r, t)$ respectively are given by [16]:

$$
r_{12}=\frac{\mu_{h} k_{1 y}-\mu_{1} k_{2 y}}{\mu_{h} k_{1 y}+\mu_{1} k_{2 y}}, t_{12}=\frac{2 \mu_{h} k_{1 y}}{\mu_{h} k_{1 y}+\mu_{1} k_{2 y}}
$$

For the other interfaces the Fresnel coefficients are:

$$
r_{23}=\frac{G k_{2 y}-\mu_{h} K}{G k_{2 y}+\mu_{h} K}, \quad t_{23}=\frac{2 G k_{2 y}}{G k_{2 y}+\mu_{h} K} \quad r_{34}=\frac{\mu_{4} K-G k_{4 y}}{\mu_{4} K+G k_{4 y}}, \quad t_{34}=\frac{2 \mu_{4} K}{\mu_{4} K+G k_{4 y}}
$$

where $G=\left(\mu_{y y} k_{3 y}+\mu_{x y} k_{3 x}\right), K=\mu_{v} \mu_{y y}, k_{1 y}=k_{4 y}, \mu_{1}=\mu_{4}=\mu_{0}$

The reflection and transmission coefficients $r^{\prime}$ and $t^{\prime}$ respectively of the structure are [17]:

$$
\begin{gathered}
r^{\prime}=\frac{B_{1}}{A_{1}}=\frac{r_{12}+r_{12} r_{23} r_{34} \mathrm{e}^{i 2 k_{3 y} d_{3}}+r_{23} \mathrm{e}^{i 2 k_{2 y} d_{2}}+r_{34} \mathrm{e}^{i 2\left(k_{2 y} d_{2}+k_{3 y} d_{3}\right)}}{1+r_{23} r_{34} \mathrm{e}^{i 2 k_{3 y} d_{3}}+r_{12} r_{23} \mathrm{e}^{i 2 k_{2 y} d_{2}}+r_{12} r_{34} \mathrm{e}^{i 2\left(k_{2 y} d_{2}+k_{3} d_{3}\right)}} \\
t^{\prime}=\frac{A_{4}}{A_{1}}=\frac{t_{12} t_{23} t_{34} \mathrm{e}^{i\left(k_{2 y} d_{2}+k_{3 y} d_{3}\right)}}{1+r_{23} r_{34} \mathrm{e}^{i 2 k_{3 y} d_{3}}+r_{12} r_{23} \mathrm{e}^{i 2 k_{2 y} d_{2}}+r_{12} r_{34} \mathrm{e}^{i 2\left(k_{2 y} d_{2}+k_{3} d_{3}\right)}}
\end{gathered}
$$


The reflectance $R$ and transmittance $T$ of the structure are given by:

$$
R=r^{\prime} r^{\prime *}, T=\left(\frac{k_{4 y}}{k_{1 y}}\right) t^{\prime} t^{\prime *}
$$

where $r^{\prime *}$ and $t^{\prime *}$ are the conjugate of $r^{\prime}$ and $t^{\prime}$ respectively.

The law of conservation of energy is [17]:

$$
R+T=1
$$

In this work, two cases of LHM are considered. The first when the incident electromagnetic waves in the visible spectral band and other one in microwave band. The frequency-dependent permittivity of LHM in the visible band is described by Drude medium model as [18]

$$
\varepsilon(\omega)=\varepsilon_{\text {lattice }}-\frac{\omega_{p}^{2}}{\omega^{2}+i \omega \gamma}
$$

where $\omega$ is the angular frequency, $\varepsilon_{\text {lattice }}$ is the lattice permittivity, $\omega_{p}$ is the effective plasma frequency and $\gamma$ is the electric damping factor.

For microwave region, I employ a dispersive LHM with $\varepsilon_{h}(\omega)$ and $\mu_{h}(\omega)$ appeared in [15] [19] as:

$$
\begin{gathered}
\varepsilon_{h}(\omega)=1-\frac{F_{e} \omega_{e p}^{2}}{\omega^{2}-\omega_{e o}^{2}+i \omega \gamma_{c}} \\
\mu_{h}(\omega)=1-\frac{F_{m} \omega_{m p}^{2}}{\omega^{2}-\omega_{m o}^{2}+i \omega \gamma_{m}}
\end{gathered}
$$

where $\omega_{e p}$ and $\omega_{m p}$ are the electric and magnetic plasma frequencies. $\omega_{e o}$ and $\omega_{m o}$ are the electric and magnetic resonance frequencies. $F_{e}$ and $F_{m}$ are the scaling filling parameters, $\gamma_{c}$ and $\gamma_{m}$ are the electric and magnetic dissipation factors.

\section{Numerical Results and Discussion}

The parameters were used in carrying out the numerical calculations are [12]: the applied field $H_{0}=0.8 \mathrm{kG}$, $m_{0}=0.56 \mathrm{kG}, H_{a}=200 \mathrm{kG}, H_{e}=540 \mathrm{kG}, \gamma=1.97 \times 10^{7} \mathrm{rad} / \mathrm{sec}$ and $\varepsilon_{1}=5.5$ for antiferromagnetic layers, $\varepsilon_{2}=8$ for the non-magnetic layers. The relative permeability of the dielectrics is $\mu_{1}=\mu_{4}=\mu_{0}$, the refractive index of the dielectrics $n_{1}=n_{4}=n_{d}$ and $d_{2}=d_{3}=d$.

\subsection{In Visible Spectral Band}

The parameters were used are [18]: $\omega_{p}=1.2 \times 10^{16} \mathrm{rad} / \mathrm{s}, \quad \gamma=1.2 \times 10^{14} \mathrm{rad} / \mathrm{s}, \quad \varepsilon_{\text {lattice }}=9.1$.

The relative permeability of LHM is assumed to be -1 . The thickness of each slab is assumed to be one-half long of the central wavelength. The reflected, transmitted power of the structure is calculated as a function of wavelength of the incident waves, angle of incidence, layer thickness and magnetic fraction of LANS. According to (16) the real part of refractive index of LHM is negative in the wavelength range of $(500,600,700,1000)$ $\mathrm{nm}$ where the real part of $n_{2}$ of values $(-1.023,-2.34,-3.27$, to -5.596$)$ where the damping factor of LHM in this region is ignored and no energy loss is displayed. The central wavelength is assumed to be $600 \mathrm{~nm}$. This choice is based on the spectral stability of the coating and for low reflectance. Stability means that the low-reflectance spectrum changes very slightly with refractive index variations as shown by Figure 2. It displays the reflected, transmitted power as a function of the normal incident wavelength when the dielectric refractive index $n_{d}$ changes to the values of $(2.34,4.86,6.58)$. It shows maximum reflectance $R$ of value $<0.25$ and minimum transmittance $T$ of value $>0.75$ at $n_{d}$ of value 2.34 over a wide wavelength range ( $\left.\lambda=500-1000 \mathrm{~nm}\right)$. The refractive indices of LHM are $(-2.34,-4.86,-6.58)$ at incident wavelength $\lambda$ of values $(600,900,1140) \mathrm{nm}$ respectively and that of LANS is $\left(n_{3}=2.49\right.$ at $\lambda=600 \mathrm{~nm}$ and magnetic fraction $\left.f_{1}=0.7\right) . n_{3}$ changes very slightly with frequency. For these indices, one minimum appears around $\lambda=600 \mathrm{~nm}$ at which the refractive indices of LHM, and LANS, dielectrics are approximately closed to each other and opposite in signs which confirm that high transmitted power can be achieved if $n_{1}=n_{4}, n_{3} \approx\left|n_{2}\right|, d_{2}=d_{3}$ [11]. These conditions which 


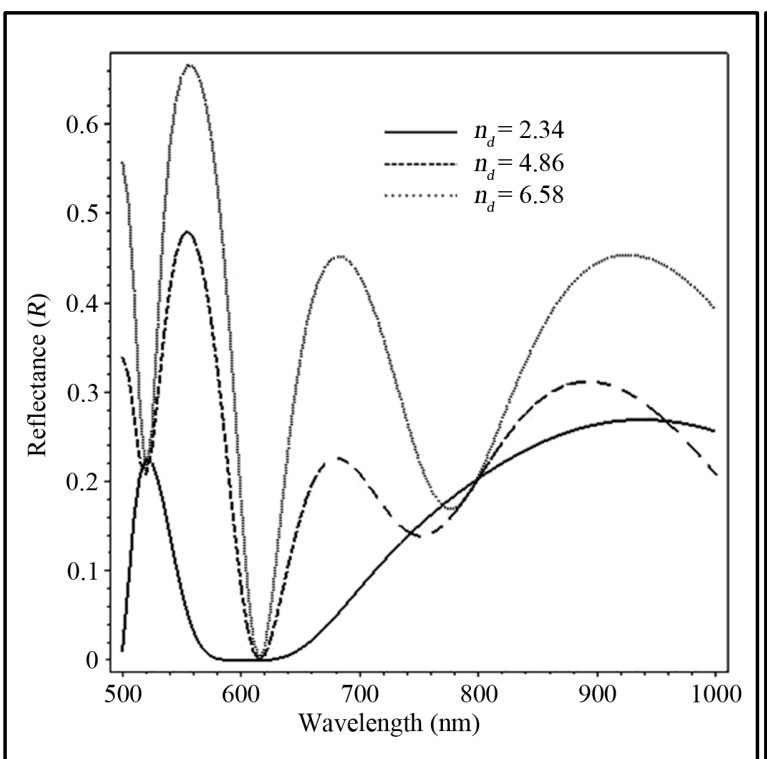

(a)

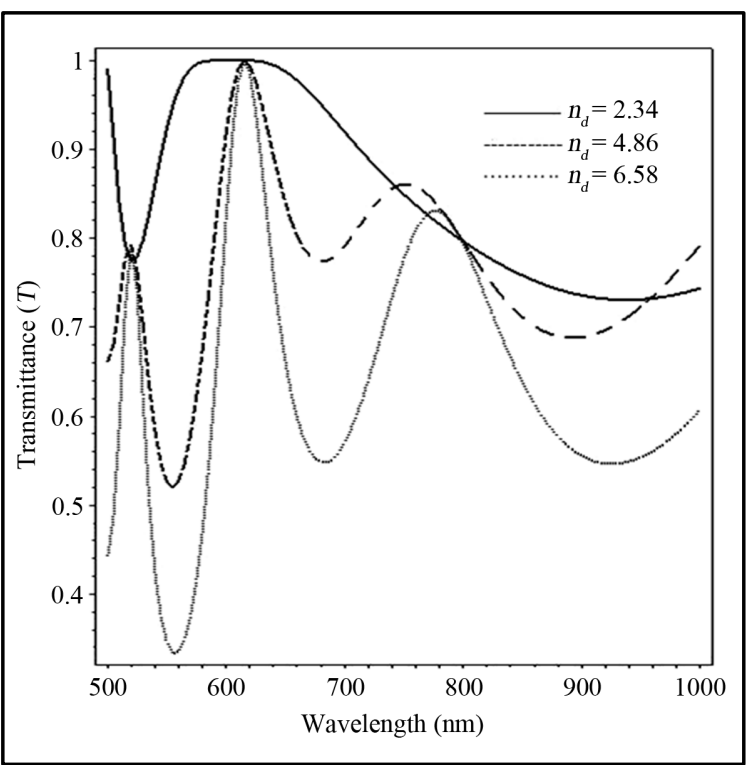

(b)

Figure 2. (a) The reflected, (b) transmitted power as a function of the normal incident wavelength when the dielectric refractive index $n_{d}$ changes as $n_{d}=2.34,4.86,6.58, f_{1}=0.7, d=250 \mathrm{~nm}$.

leads to $R=0$ and $T=1$ around $\lambda=600 \mathrm{~nm}$ are: $r_{23}=0, t_{23}=1, k_{2 y}=-k_{3 y}=0.3498 \times 10^{8} \mathrm{~m}^{-1}$, $r_{12}=-r_{34}=0.27225, t_{12}=1.27225, t_{34}=0.728, t_{12} t_{34}=1+r_{12} r_{34}=0.926$. Such AR coating systems are used in photodiodes (LASER) and other optoelectronic devices which need a minimum reflectance at a single wavelength. Figure 3 illustrates the reflectance spectra over a wide range of incidence angle for $\lambda$ of values (600, 700, $800) \mathrm{nm}$. In the range $\left(\theta=0^{\circ}, 27^{\circ}\right)$ the minimum reflectance is achieved at $\lambda=600 \mathrm{~nm}$. The implementation of LANS layer adjacent to LHM layer dramatically reduces the reflection and greatly enhances the transmission near a specifically frequency at incidence angles $\left(\theta=0^{\circ}, 27^{\circ}\right)$. By wavelength increase to the value of $800 \mathrm{~nm}$, minimum reflectance is observed at higher incidence angle of value $57^{\circ}$. The effect of the magnetic fraction $f_{1}$ on the reflectance and transmittance is described in Figure 4. As $f_{1}$ increases to the values of $(0.1,0.5,0.9)$, the refractive index of LANS $n_{3}$ decreases to the values of $(2.78,2.59,2.39)$ while $n_{2}$ of LHM increases to the value of $(-2.83$ to -4.486$)$ in the wavelength range $(600-850) \mathrm{nm}$ which leads to reflectance increase. It is worth to note that, $R=0$ and $T=1$ at $\lambda=600 \mathrm{~nm}, f_{1}=0.9$. This is because $n_{1}=n_{4}=4.86, \quad n_{3} \approx\left|n_{2}\right|=2.39$, $d_{2}=d_{3}$. Figure 5 demonstrates the effect of thickness $d$ of LHM on the reflectance and transmittance at normal incidence of $\lambda=600 \mathrm{~nm}, 800 \mathrm{~nm}$. The slab thickness is changed from $50 \mathrm{~nm}$ to $500 \mathrm{~nm}$. It is noticed that reflectance and transmittance changes periodically with thickness. Besides that, more transmittance is realized at $\lambda=$ $800 \mathrm{~nm}$ where maximum $R$ is 0.03 while minimum $T=0.97$ of the incident power. It is shown that the selected thickness $(d=250 \mathrm{~nm})$ is appropriate for achieving zero reflectance at $\lambda=600 \mathrm{~nm}$.

\subsection{In Microwave Spectral Band}

For dispersive LHM with $\varepsilon_{h}(\omega)$ and $\mu_{h}(\omega)$ have parameters appeared in [15] as: $\omega_{m p}=2 \pi 10.95 \mathrm{GHz}$, $\omega_{m o}=2 \pi 10.1 \mathrm{GHz}, \omega_{e p}=2 \pi 13.3 \mathrm{GHz} \quad F_{e}=0.37, \omega_{e o}=2 \pi 10.3 \mathrm{GHz}, F_{m}=0.26, \gamma_{c}=\gamma_{m}=0.1$. For this LHM, the frequency range in which $\varepsilon_{h}(\omega)$ and $\mu_{h}(\omega)$ are negative extends from 10.4 up to $11.5 \mathrm{GHz}$ with corresponding wavelength extends from 26 up to $28 \mathrm{~mm}$. The thickness of each of LHM and dielectrics slabs is equal to one half-wavelength long at the operating frequency. Reflectance and transmittance are calculated numerically as stated above. The law of conservation of energy is [20]:

$$
R+T=1-P_{\text {loss }}
$$

where $P_{\text {loss }}$ is the loss power due to losses in LHM. Since in the structure arrangement shown in Figure 1, there is no effect of magnetic fraction of LANS on the reflected power and there is very small power loss of LHM which can't be displayed in a figure, I rearranged the structure to be LANS in Region 1 instead of the 


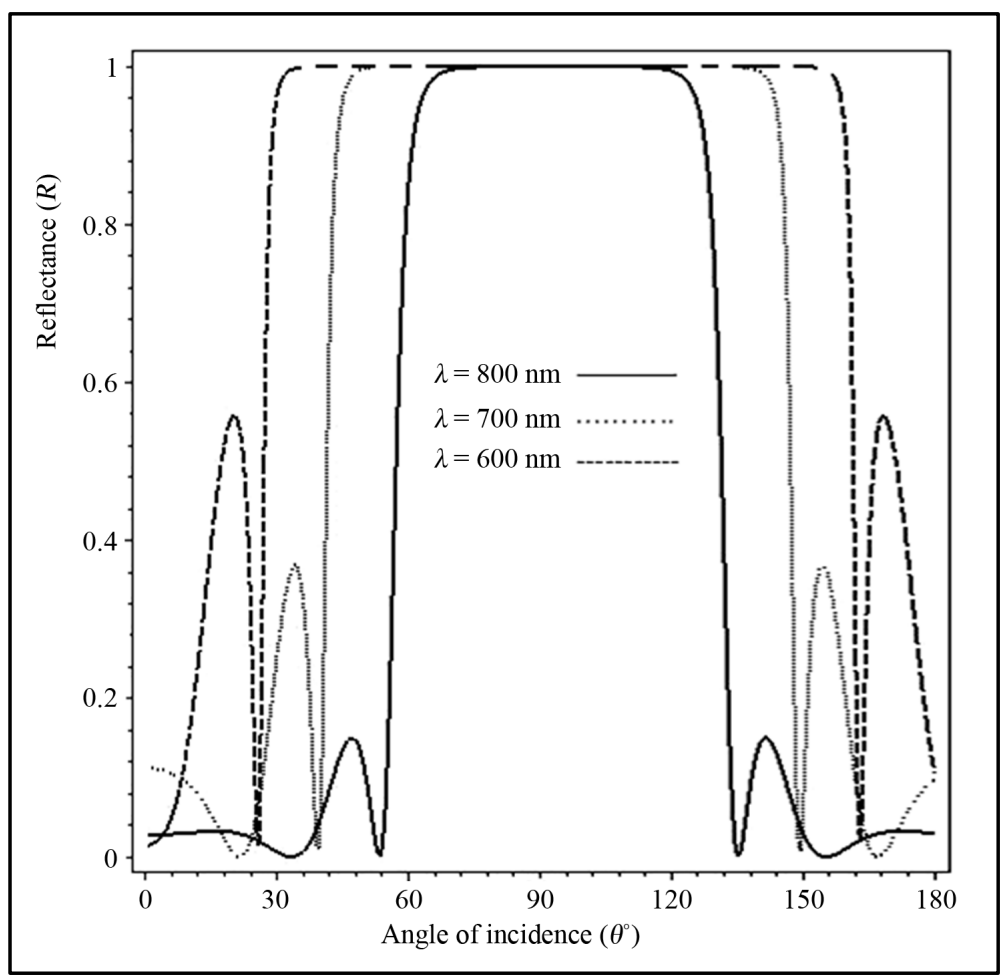

Figure 3. The reflected power as a function of the angle of incidence for different wavelength $\lambda=600,700,800 \mathrm{~nm}, n_{d}=4.86, f_{1}=0.7, d=250 \mathrm{~nm}$.

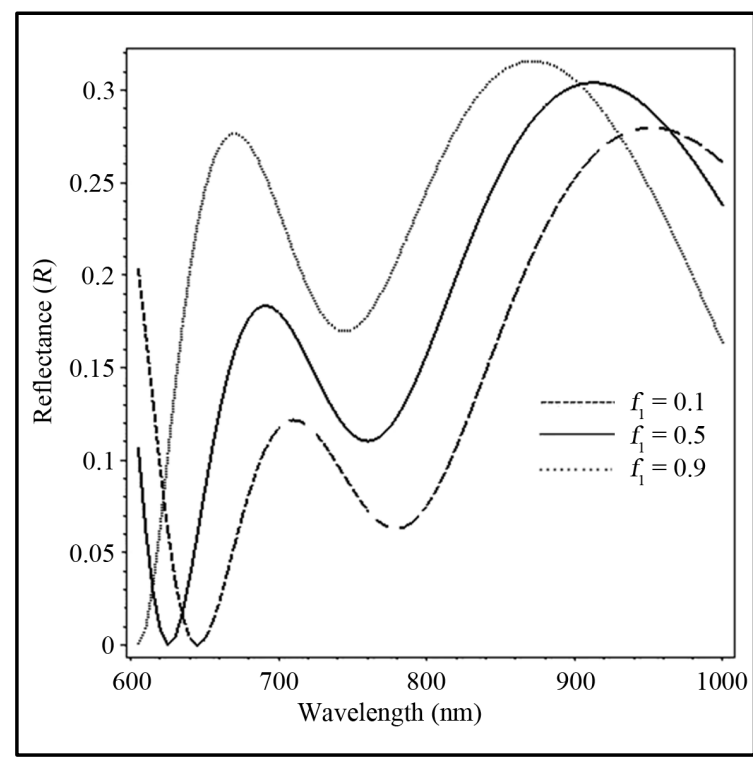

(a)

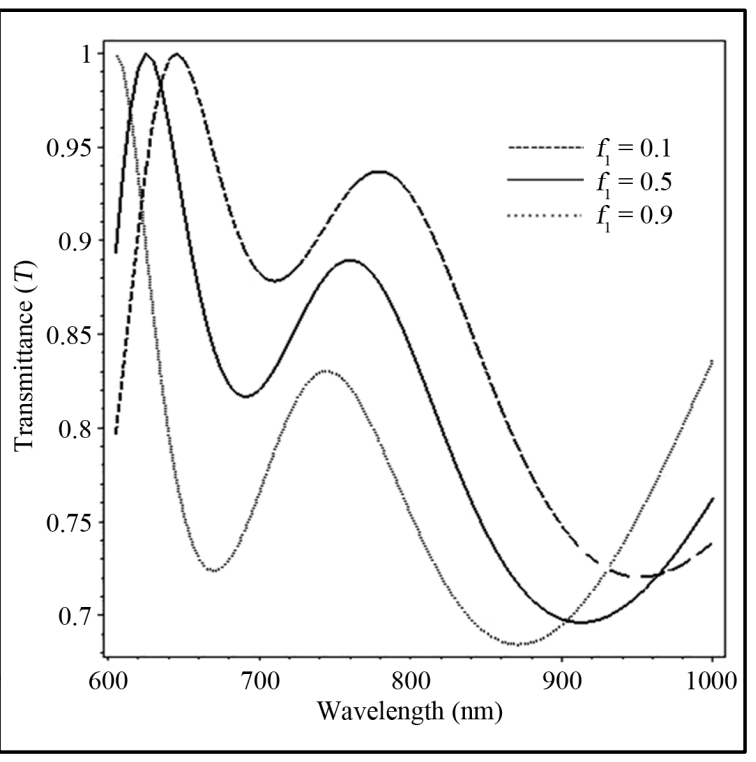

(b)

Figure 4. (a) The reflected, (b) transmitted power versus the normal incident wavelength when the magnetic fraction $f_{1}$ changes as $f_{1}=0.1,0.5,0.9, n_{d}=4.86, d=250 \mathrm{~nm}$.

dielectric which will be in Region 3. The reflection, transmission coefficients are rewritten as:

$$
r_{12}=\frac{\mu_{h}\left(\mu_{y y} k_{1 y}+\mu_{x y} k_{1 x}\right)-\mu_{v} \mu_{y y} k_{2 y}}{\mu_{h}\left(\mu_{y y} k_{1 y}+\mu_{x y} k_{1 x}\right)+\mu_{v} \mu_{y y} k_{2 y}},
$$




$$
t_{12}=\frac{2 \mu_{h}\left(\mu_{y y} k_{1 y}+\mu_{x y} k_{1 x}\right)}{\mu_{h}\left(\mu_{y y} k_{1 y}+\mu_{x y} k_{1 x}\right)+\mu_{v} \mu_{y y} k_{2 y}}
$$

For the other interfaces the Fresnel coefficients are:

$$
\begin{aligned}
& r_{23}=\frac{\mu_{3} k_{2 y}-\mu_{h} k_{3 y}}{\mu_{3} k_{2 y}+\mu_{h} k_{3 y}}, \quad t_{23}=\frac{2 \mu_{3} k_{2 y}}{\mu_{3} k_{2 y}+\mu_{h} k_{3 y}} \\
& r_{34}=\frac{\mu_{4} k_{3 y}-\mu_{3} k_{4 y}}{\mu_{4} k_{3 y}+\mu_{3} k_{4 y}}, \quad t_{34}=\frac{2 \mu_{4} k_{3 y}}{\mu_{4} k_{3 y}+\mu_{3} k_{4 y}}
\end{aligned}
$$

Figure 6 displays the reflectance, transmittance and power loss as a function of the incident wavelength for many values of magnetic fraction. The operating wavelength is assumed to be $0.027 \mathrm{~m}$ which is included in the frequency range in which $\varepsilon_{h}(\omega)$ and $\mu_{h}(\omega)$ are simultaneously negative. As $f_{1}$ increases to the values of $(0.1,0.5,0.9) n_{1}$ of LANS decreases to the values of $(2.78,2.59,2.39)$ respectively, $n_{2}$ of LHM changes to the values $\left(0.04-i 0.17 \times 10^{-9}\right)$ to $\left(1.37-i 0.53 \times 10^{-10}\right)$ in the wavelength range of 0.026 to $0.272 \mathrm{~m} . R=0$ and $T$ $=1$ and zero power dissipation are attained at wavelength range $\lambda=0.026-0.272 \mathrm{~m}$ and $f_{1}=0.1$ at $\theta=30^{\circ}$.

\section{Conclusions}

The transmission and reflection of perpindicular polarized waves by a multilayered structure consisting of a pair of LHM and LANS materials embedded between two semi-infinite dielectrics media have been studied in both visible and microwave spectral bands with the appropiate LHM and appropiate location of LANS in the structure. The frequency dependence of $\varepsilon_{h}(\omega)$ and $\mu_{h}(\omega)$ of LHM and that of LANS is taken into account. It has been shown that, the frequency-dependent refractive index of both LHM and LANS plays an important role in the variation of the reflection coefficients of the structure. Low reflection can be achieved for both visible and microwave rays by choosing the proper indicies of the materials constiuted the structure. For incident visible rays, LANS is located as shown in Figure $1 R=0$ and then $T=1$ is attained at a sigle wavelength value of 600 $\mathrm{nm}$ with magnetic fraction of LANS of value 0.9 , incidence angle of value $0^{\circ}$ or $27^{\circ}$. For incident microwave rays, LANS is located in Region 1 and the dielectric in Region 3. $R=0$ and then $T=1$ are achieved at wavelength

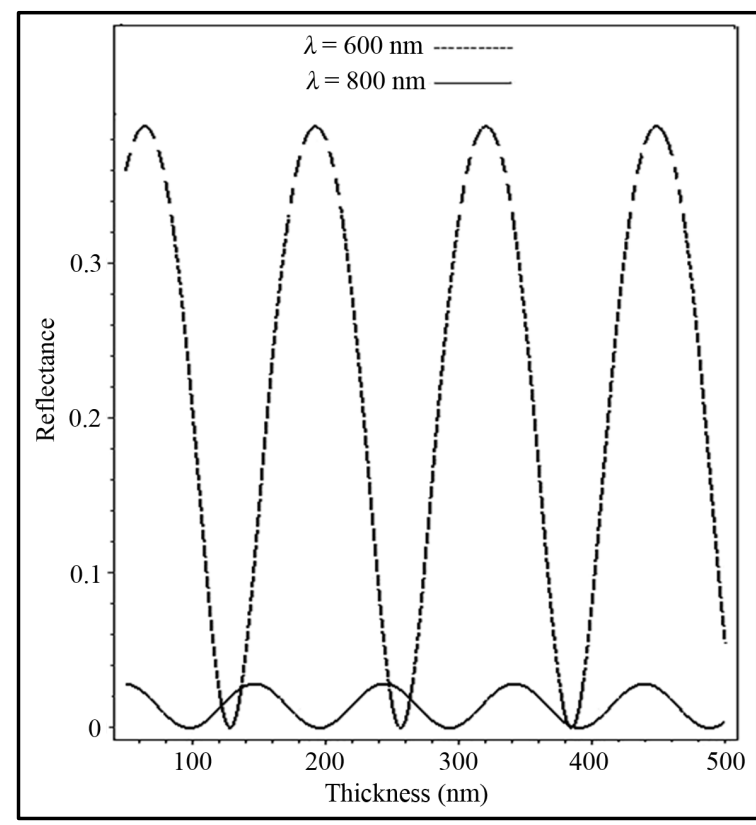

(a)

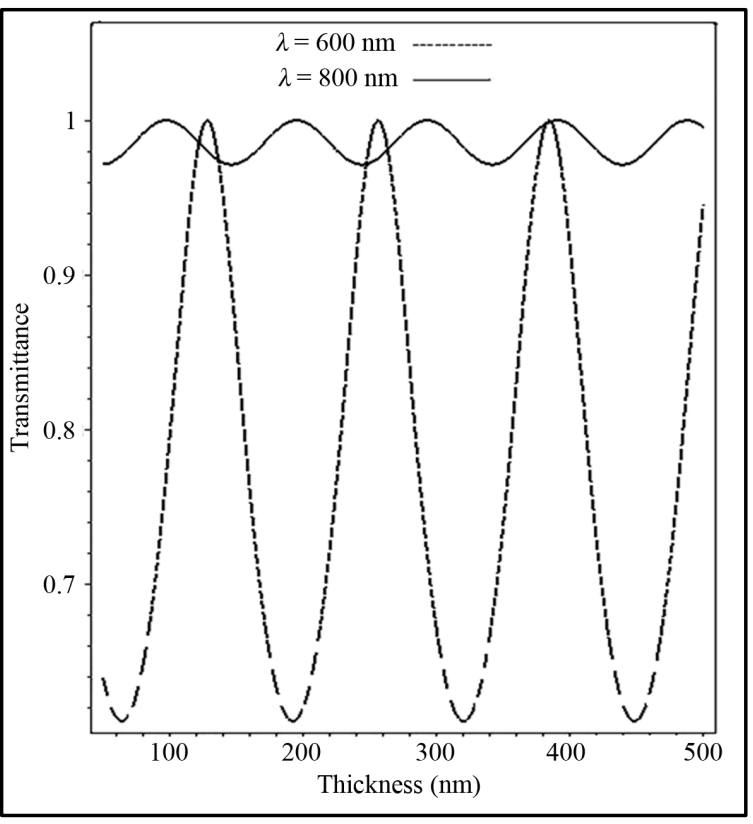

(b)

Figure 5. (a) The reflected, (b) transmitted power versus the layer thickness $d$ at normal incident wavelength of $\lambda=600$ $\mathrm{nm}, 800 \mathrm{~nm}, n_{d}=4.86, f_{1}=0.7$. 


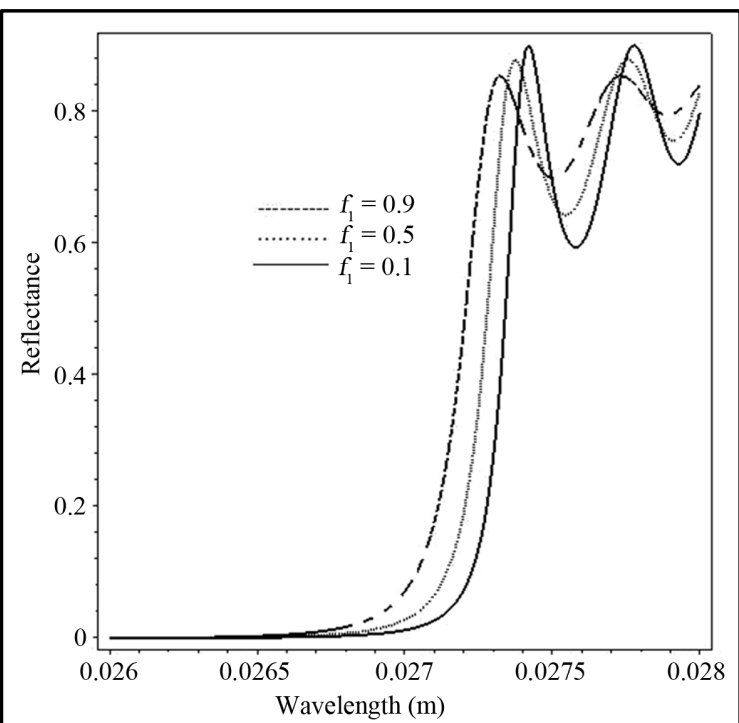

(a)



(b)

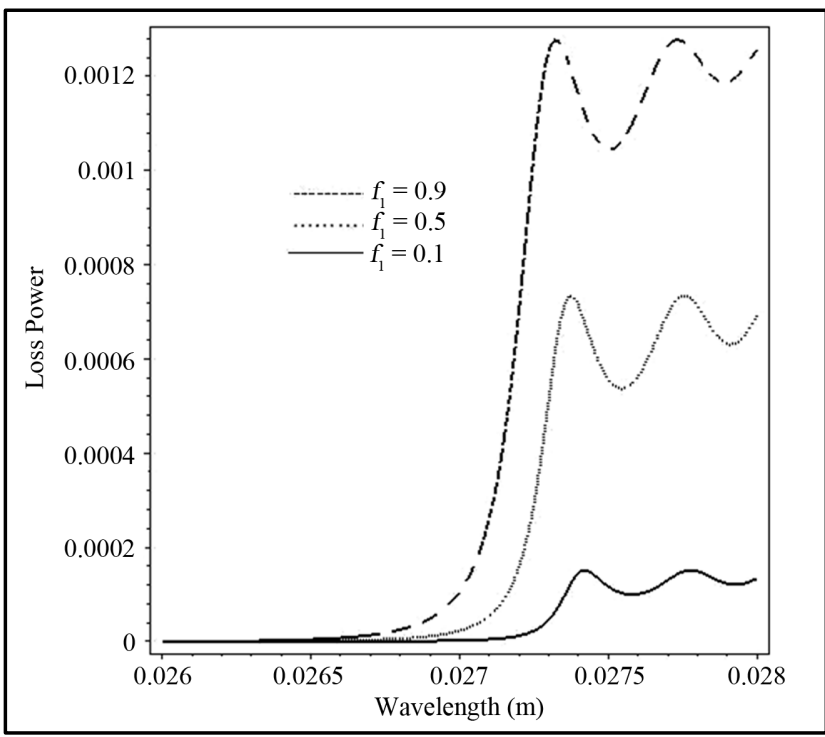

(c)

Figure 6. (a) The reflected, (b) transmitted, (c) loss power versus the incident wavelength when the magnetic fraction $f_{1}$ changes as $f_{1}=0.1,0.5,0.9, n_{d}=4.86, \theta=30^{\circ}, d=14 \mathrm{~mm}, \gamma_{e}=\gamma_{m}=0.1$

$\lambda=0.026$ to $0.272 \mathrm{~m}$ with $f_{1}=0.1$ at $\theta=30^{\circ}$. The implementation of LANS adjacent to LHM layer dramatically reduces the reflection and greatly enhances the transmission near a specifically frequency. The law of conservation of energy has been satisfied by the obtained results. The obtained results may be used to refine the understanding of any related applications that may be modeled requiring controlling of reflected and transmitted powers as photovoltaic cells and optoelectronic devices.

\section{References}

[1] Dobrowolski, J.A., Poitras, D., Ma, P., Vakil, H. and Acree, M. (2002) Applied Optics, 41, 3075-3083. http://dx.doi.org/10.1364/AO.41.003075

[2] Deinega, A., Valuev, I., Potapkin, B. and Lozovik, Y. (2011) JOSA A, 28, 770-777.

[3] Bouhafs, D., Moussi, A., Chikouche, A. and Ruiz, M. (1998) Solar Energy Materials and Solar Cells, 52, 79-93.

[4] Chen, H., Zhou, J., Hara, J., Chen, F., Azad, A.K. and Taylor, A.K. (2010) Physical Review Letters, 105, Article ID: 
073901. http://dx.doi.org/10.1103/PhysRevLett.105.073901

[5] Mousa, H.M. and Shabat, M.M. (2012) Applied Physics A, 111, 1057-1063. http://dx.doi.org/10.1007/s00339-012-7482-5

[6] Mousa, H.M. and Shabat, M.M. (2011) International Journal of Modern Physics B, 25, 4543. http://dx.doi.org/10.1142/S0217979211052071

[7] Sopori, B.L. and Pryor, R.A. (1983) Solar Cells, 8, 249-261. http://dx.doi.org/10.1016/0379-6787(83)90064-9

[8] Redfield, D. (1981) Solar Cells, 3, 27-33. http://dx.doi.org/10.1016/0379-6787(81)90079-X

[9] Buie, D., McCann, M.J., Weber, K.J. and Dey, C.J. (2004) Solar Energy Materials and Solar Cells, 81, 13-24. http://dx.doi.org/10.1016/j.solmat.2003.08.009

[10] Oraizi, H. and Afsahi, M. (2009) Progress in Electromagnetics Research B, 14, 263-283.

[11] Cory, H. and Zach, C. (2004) Microwave and Optical Technology Letters, 40, 460-465. http://dx.doi.org/10.1002/mop.20005

[12] Shabat, M.M. and Mousa, H.M. (2007) SPIE Proceedings, 6582.

[13] Vigoureux, J.M. (1991) Journal of the Optical Society of America, 8, 1697-1701.

[14] Kong, J.A. (2005) Theory of Electromagnetic Waves. EMW Publishing.

[15] Shelby, R.A. (2001) Microwave Experiments with Left-Handed Materials. Ph.D. Thesis, University of Calefornia, San Diego.

[16] Caloz, C. and Itoh, T. (2006) Electromagnetic Metamaterials. John Wiley and Sons, New Jersey.

[17] Kong, J.A. (2002) Progress in Electromagnetics Research-PIER, 35, 1-52. http://dx.doi.org/10.2528/PIER01082101

[18] Isaac, T.H. (2009) Tunable Plasmonic Structures for Terahertz Frequencies. Ph.D. Thesis, University of Exeter, Exeter.

[19] Pendry, J.B., Holden, A.J., Sewart, W. and Youngs, I. (1996) Physical Review Letters, 76, 4773-4776. http://dx.doi.org/10.1103/PhysRevLett.76.4773

[20] Cory, H. and Zach, C. (2004) Microwave and Optical Technology Letters, 40, 460-465. http://dx.doi.org/10.1002/mop.20005 